\title{
Gene coexpression network analysis identifies genes and biological processes shared among anterior pituitary and brain areas that affect estrous behavior in dairy cows
}

\author{
A. Kommadath, ${ }^{1}$ M. F. W. te Pas, and M. A. Smits ${ }^{2}$ \\ Animal Breeding and Genomics Centre, Wageningen UR Livestock Research, PO Box 65, 8200 AB, Lelystad, the Netherlands
}

\begin{abstract}
The expression of estrous (sexually receptive) behavior (EB), a key fertility trait in dairy cows, has been declining over the past few decades both in intensity and duration. Improved knowledge of the genomic factors underlying EB, which is currently lacking, may lead to novel applications to enhance fertility. Our objective was to identify genes and biological processes shared among the bovine anterior pituitary (AP) and four brain areas that act together to regulate EB by investigating networks of coexpressed genes between these tissues. We used a systems biology approach called weighted gene coexpression network analysis for defining gene coexpression networks using gene expression data from the following tissues collected from 14 cows at estrus: AP, dorsal hypothalamus (DH), ventral hypothalamus (VH), amygdala (AM), and hippocampus (HC). Consensus modules of coexpressed genes were identified between the networks for the AM-DH, HC-DH, VH$\mathrm{DH}, \mathrm{AP}-\mathrm{DH}$, and AM-HC tissue pairs. The correlation between the module's eigengene (weighted average gene expression profile) and levels of EB exhibited by the experimental cows were tested. Estrous behavior-correlated modules were found enriched for gene ontology terms like glial cell development and regulation of neural projection development as well as for Kyoto Encyclopedia of Genes and Genomes pathway terms related to brain degenerative diseases. General cellular processes like oxidative phosphorylation and ribosome and biosynthetic processes were found enriched in several correlated modules, indicating increased transcription and protein synthesis. Stimulation of ribosomal RNA synthesis is known from rodent studies to be a primary event in the activation of neuronal cells and pathways involved in female reproductive behavior and this pre-
\end{abstract}

Received June 6, 2012.

Accepted November 19, 2012.

${ }^{1}$ Present address: Alberta Ingenuity Centre for Livestock Genomics Technology, University of Alberta, 1400 College Plaza, 8215 - 112 Street, Edmonton, Canada T6G 2C8

${ }^{2}$ Corresponding author: mari.smits@wur.nl cedes the estrogen-driven expansion of dendrites and synapses. Similar processes also operate in cows to affect EB. Hub genes within EB-correlated modules (e.g. NEFL, NDRG2, GAP43, THY1, and TCFYL2, among others) are strong candidates among genes regulating EB expression. The study improved our understanding of the genomic regulation of EB in dairy cows by providing new insights into genes and biological processes shared among the bovine AP and brain areas acting together to regulate EB. The new knowledge could lead to the development of novel management strategies to monitor and improve reproductive performance in dairy cows (for example, biomarkers for estrus detection).

Key words: brain, dairy cow, estrous behavior, gene coexpression

\section{INTRODUCTION}

Dairy cow fertility is a trait of economic importance to dairy farmers and the dairy industry; therefore, losses due to subfertility or infertility are of major concern (Stott et al., 1999). Currently, we have a basic understanding of the physiological mechanisms that influence the multifactorial trait of fertility (Garnsworthy et al., 2008), especially the hormones involved and the link with nutrition and energy balance (Butler, 2000). At the genomic level, gene expression studies on tissues and organs involved in female reproductive processes, embryo development and implantation, and maintenance of pregnancy have helped to identify genes and biological processes related to fertility (Ushizawa et al., 2004; Bauersachs et al., 2005; Casey et al., 2005; Salilew-Wondim et al., 2010). However, there is a lack in our understanding of the genomic factors underlying bovine estrous behavior (EB), a key fertility trait shown when cows are sexually receptive. The expression of EB, which is regulated by the brain and pituitary, has been declining both in intensity and duration over the past few decades along with the decline in several other fertility traits (Lopez et al., 2004). The proper expression of EB is important because it helps farmers to identify the fertile period in the cows' estrous cycles 
when AI has the greatest chance to result in successful fertilization.

In an effort to understand the genomic regulation of $\mathrm{EB}$, we studied gene expression in the anterior pituitary (AP) and 4 brain areas: dorsal hypothalamus (DH), ventral hypothalamus (VH), amygdala (AM), and hippocampus (HC) at 2 time points of the cow's estrous cycle: start of estrus (d 0) and mid-cycle (d 12). The choice of these tissues was motivated by the fact that the anterior pituitary and hypothalamus are parts of the hypothalamic-pituitary-ovarian axis and important centers controlling the female reproductive cycle and behavior (Mong and Pfaff, 2004; Pfaff, 2005), whereas the amygdala and hippocampus have functions related to sexual behavior and associated emotional responses (Gallagher and Chiba, 1996; Salamon et al., 2005). In a previous study (Kommadath et al., 2010), we focused on the association between gene expression profiles of the aforementioned tissues and the trait of showing EB, as quantified by so-called heat scores. In all 5 tissues, we identified sets of genes whose expression was associated with heat scores. However, in real-life scenarios, the expression of a complex trait like behavior is almost certainly the result of intricate interactions between different tissues in response to hormonal, visual, physiological, and other internal and external signals. Therefore, studying gene coexpression patterns among the AP and brain areas may shed light on biological processes for which these tissues act together in regulating EB expression. Furthermore, by virtue of the greater power of the coexpression analyses among tissue pairs compared to analyses at the level of individual tissue samples, additional EB-associated genes may be expected to be revealed here. Microarray studies have shown that clusters of genes with similar expression profiles across samples may form part of a specific multiprotein complex or biological pathway or are participants in a specific regulatory or signaling circuit (Eisen et al., 1998; Ideker et al., 2002; Huang et al., 2007; Horvath and Dong, 2008). Specifically, in a study of brain gene expression data, it was shown that the transcriptomes of human brain regions were robustly organized into modules of coexpressed genes that reflect their underlying cellular composition (Oldham et al., 2008). Here, modules refer to clusters of highly interconnected genes within a gene coexpression network. Other studies used coexpression networks to screen for genes underlying complex traits (for example, BW; Ghazalpour et al., 2006). Therefore, gene coexpression networks constructed from gene expression data can effectively capture relationships between transcripts, which group as modules that reveal biologically meaningful higher-order organization of the transcriptome (Langfelder and Horvath, 2007).
The objective of this study was to identify genes and biological processes shared among the bovine AP and brain areas that affect EB by investigating networks of coexpressed genes between these tissues. Studying these coexpressed genes may shed light on how these tissues act together to regulate EB.

\section{MATERIALS AND METHODS}

\section{Phenotypic Data Recording and Gene Expression Measurements}

The microarray experiment was carried out as part of a study aimed at identifying and studying genes that contribute to differences in EB expression and fertility levels of dairy cows. The procedures followed for EB recording, tissue collection, RNA extraction, and microarray hybridization were as described in our earlier studies (Kommadath et al., 2010, 2011). The recording of milk progesterone levels combined with ultrasonography of the ovarian structures helped identify cows approaching the estrus phase. Briefly, EB was recorded in 28 healthy Holstein-Friesian cows from 30 DIM onwards until their time of euthanization, which varied between 77 and 139 DIM (i.e., after at least 2 estrous cycles). The cows were observed daily for the signs of $\mathrm{EB}$ during 2 observation periods of $30 \mathrm{~min}$ each: one in the morning (after milking but before feeding) and one in the evening (after milking and feeding). EB scores per 30-min observation period were calculated by adding up the recorded occurrence of specific behaviors weighted as per the scoring protocol described in Kommadath et al. (2010). The EB scores from multiple consecutive cycles per cow were averaged to obtain the average heat score per cow. The final confirmation that the cow had entered its estrus phase prior to euthanization was based on the additional evidence of EB display. The cows were euthanized in a stress-free, quick and standardized way and all efforts were made to minimize suffering. The study was approved by the Animal Care and Ethics Committee of the Animal Sciences Group of Wageningen University and Research Centre (Lelystad, the Netherlands; approval ID 2006087a). Samples from 4 brain areas (DH, VH, AM, and $\mathrm{HC}$ ) and the $\mathrm{AP}$ were collected from these cows, 14 of which were euthanized at start of estrus (d 0) and 14 in the middle of the estrous cycle (d 12). To ensure that exactly the same areas of the brain were sampled from the different cow brains, the position of the whole brain samples were fixed in a special box and defined slices were made. Defined cuts of the slices were then made to get the specific samples. The procedure followed for collecting brain samples is described in detail in Additional file 4 of Kommadath et al. (2011). It may be noted that 
the sampled brain areas are composed of heterogeneous cell populations and different brain nuclei. The RNA extracted from tissue samples were hybridized on Bovine 24K (24,000) oligonucleotide (70-mer) microarrays designed and produced by the Bovine Oligonucleotide Microarray Consortium (BOMC; http://www. bovineoligo.org/). A total of 280 arrays (i.e. 28 cows $\times$ 5 tissues $\times 2$ for dye swaps) were prepared in a common reference design, with the dye labels swapped between individual samples from each tissue and a reference sample consisting of equal proportions of RNA from all tissues of all cows. Microarray data preprocessing and analysis were done using the linear models for microarray data (LIMMA) package (Smyth, 2005) within the Bioconductor project (Gentleman et al., 2004) of the $\mathrm{R}$ statistical programming language (http://www. r-project.org). Gene expression levels, expressed as Mvalues (log differential-expression ratio of sample versus reference), were thus obtained for all probes per array.

The array design (accession number: A-MEXP-1781) and the array experiment data (accession number: ETABM-916) are publicly accessible on ArrayExpress (http://www.ebi.ac.uk/microarray-as/ae/). The original annotation of the bovine $24 \mathrm{~K}$ oligonucleotide microarray provided by the BOMC dates back to June 2007 . For our analysis, we used the bovine oligonucleotide array probe reannotation (version 5) based on Ensembl (http://www.ensembl.org) release 56 (October 2009) provided on the European Animal Disease Genomics Network of Excellence (EADGENE) website (http: //www.eadgene.info/ToolsResources/EADGENE OligoSetsAnnotationFiles/tabid/324/Default.aspx) by the authors of the oligo-set reannotation pipeline sigReannot (Casel et al., 2009). In the sigReannot pipeline, probes on the bovine oligonucleotide array were first checked for their specificity by aligning them to all known bovine transcripts. The reannotation was limited to those 16,620 of the 23,496 probes (excluding control probes) that could be linked to a unique bovine gene.

For the analysis done here, we used only the data from the d-0 cows (start of estrus) with the objective to investigate networks of coexpressed genes that correlated to EB scores. The M-values of genes represented by multiple probes were averaged, leaving gene expression data on 13,234 unique genes per array. The various steps taken in the analysis are summarized in Table 1.

\section{Construction of Gene Coexpression Networks and Identification of Consensus Modules Among Pairs of Tissues}

The hypothalamus is the master regulatory center within the hypothalamic-pituitary-ovarian axis that responds to hormonal signals and stimulates the AP to release hormones by secreting releasing factors and

Table 1. Summary of steps taken in the analysis and their results

Steps
Process data of 14 pairs of dye-swapped microarrays per tissue
collected from 14 cows at start of estrous cycle
Select good quality probes based on probe reannotation and average
the M-values of probes representing the same gene
Select the top $50 \%$ most variable genes per tissue and identify genes
shared by each tissue within a tissue pair (assuming that genes
affecting estrous behavior expression would have a variable expression
across the experimental cows showing differing levels of estrous
behavior)
Perform coexpression network analysis on gene expression data of
shared genes within each tissue pair and identify consensus modules
Identify consensus modules within tissue pairs whose module
eigengenes correlate with estrous behavior scores

Test for enriched gene ontology and Kyoto Encyclopedia of Genes and Genomes (KEGG) pathway terms within estrous behavior-correlated consensus modules

Identify hub genes within the estrous behavior correlated consensus modules

\section{Results}

Gene expression values (M-values) of 23,496 probes per array obtained

16,620 good-quality probes per array representing 13,234 genes obtained

Approximately 4,000 to 5,000 genes per tissue pair obtained : AM$\mathrm{DH}, \mathrm{HC}-\mathrm{DH}, \mathrm{VH}-\mathrm{DH}, \mathrm{AM}-\mathrm{HC}$, and AP-DH ${ }^{1}$

Gene coexpression networks constructed for tissues within each pair and consensus modules identified

Consensus modules that correlated with estrous behavior identified: AM-DH: 1 of 3, HC-DH: 5 of 10, VH-DH: 0 of 2, AMHC: 3 of 8, and AP-DH: 10 of 23

Significant enriched terms obtained (summary of the significant terms per module are reported in Figure 1)

Hub genes per module obtained (the top 3 hub genes per module are reported in Table 3 )

\footnotetext{
${ }^{1}$ Tissues are abbreviated as follows: anterior pituitary (AP), dorsal hypothalamus (DH), ventral hypothalamus (VH), amygdala (AM), and hippocampus (HC).
} 
signaling by neural pathways. Therefore, here we chose to study gene coexpression patterns between the $\mathrm{DH}$ and each of the other tissues in this study: VH, AM, $\mathrm{HC}$, and AP. In addition, we also analyzed the AM-HC pair, as the tissues in this pair are known to be involved in emotional responses that could play a role in enhancing EB.

To begin with, the gene expression data was tested for genes and arrays having an excessive number of missing values, which, if any, were removed from further analysis. The arrays were then clustered to identify any outliers. From the 13,234 unique good-quality genes in the array, the top $50 \%$ of genes with the most variable expression among samples per tissue were selected for further analysis. For this selection, we first calculated the variance for each gene among all samples per tissue, ranked them by variance, and selected those at the top $50 \%$. The selection was done because the most interesting genes would be the ones whose differing expression contributes to the differing level of the trait's expression. Genes whose expression remains the same in all cases would generally not be associated with determining the level of the trait's expression. Further, analyzing a subset of the most interesting genes instead of taking all genes together makes the procedures involved in network construction and analysis more refined and less computationally demanding. For the gene coexpression network analysis, only those genes among the top 50\% genes that were shared between each pair of tissues under study were used.

For defining gene coexpression networks and identifying consensus modules of coexpressed genes among tissue pairs, we used a systems biology approach known as weighted gene coexpression network analysis (WGCNA; Zhang and Horvath, 2005; Langfelder and Horvath, 2008) implemented in the $\mathrm{R}$ statistical programming language (http://www.r-project.org) as a freely available software package called WGCNA. Briefly, WGCNA is a network-based gene screening method that integrates information on gene significance (correlation between gene expression and a trait of interest) and module membership to identify candidate genes. Detailed tutorials with examples for the use of this method can be found at http:// labs.genetics.ucla.edu/horvath/htdocs/Coexpression Network/Rpackages/WGCNA/Tutorials/. Pearson correlations were first calculated for all pairs of genes across all microarray samples, followed by a noisereduction step where these correlations are weighted by raising their absolute values to a certain power, $\beta$. The pickSoftThreshold function in the WGCNA package lists network properties for different choices of the power. The soft-thresholding power $\beta$ was then selected using the scale-free topology criterion, a property of most biological networks. For the resulting weighted network, gene coexpression modules were defined as branches of a hierarchical clustering tree. Specifically, we used average linkage hierarchical clustering with the topological overlap similarity measure to define a cluster tree. The topological overlap is a robust measure of interconnectedness, which keeps track of shared patterns of connection strengths. For branch cutting (module detection) we used the dynamic branch-cutting algorithm (Langfelder et al., 2008) implemented in WGCNA to detect modules larger than 20 genes. The modules that are shared between the tissues in each pair (consensus modules) were then identified and assigned unique color labels.

\section{Characterization of Consensus Modules That Correlate With Estrous Behavior}

The consensus modules whose module eigengenes correlated with EB (correlation >0.3) were identified using a function within the WGCNA package that correlates module eigengenes with trait values. The module eigengene is the most representative gene expression in the module and corresponds to the first principal component of that module. The correlation is tested by taking the module eigengenes as covariates of a multivariate regression model that regresses the trait of EB expressed as heat scores on the eigengenes transcriptome (Langfelder and Horvath, 2007).

For gaining insight into biological processes underlying $\mathrm{EB}$, we performed functional analyses of the genes within the EB correlated modules (study sets) using the R package GOstats (Falcon and Gentleman, 2007). Genes in the study sets were tested for enriched gene ontology biological process (Ashburner et al., 2000) terms and Kyoto Encyclopedia of Genes and Genomes (KEGG; Kanehisa and Goto, 2000) pathway terms (Benjamini and Hochberg, 1995; corrected P-value: $<0.15)$. Bovine Ensembl ID were used for the study sets, whereas the population set consisted of Entrez gene ID as required by the GOstats package. Of the 13,234 unique good-quality genes on the array, 12,655 remained after conversion to Entrez gene ID using the Bioconductor package org.Bt.eg.db (Carlson, 2011).

The genes in the consensus modules were ordered by their module eigengene-based intramodular connectivity measures (kME) to identify genes with the highest connectivity. The most important genes in the network are usually the most well connected with other genes and are called hub genes. As an example, we looked in detail at the known functions of the top hub genes in one of the EB-correlated consensus modules for their likely relation to EB regulation. Further, we visualized this module as a network, with the nodes represent- 
ing the genes and the edges representing the intergenic connection strengths. We used a function in WGCNA to export the genes from the weighted network data of these modules in a form that was compatible for visualization using the Cytoscape software (Shannon et al., 2003). We chose a cutoff threshold of 0.2 to filter out low-strength connections between genes in the network.

\section{RESULTS}

\section{Estrous Behavior Scores and Gene Expression Data}

For each cow, the level of EB expression was quantified as its average heat score using EB scores recorded from at least 2 consecutive cycles (Table 2). For identifying modules of coexpressed genes correlated with $\mathrm{EB}$, we adjusted the heat score of 1 of the d- 0 cows to the level of the next highest cow (405) because of its high outlier value of 1,750 , which we attributed to that cow's several consecutive attempts to mount other cows during one observation period. The average heat scores for the remaining $13 \mathrm{~d}-0$ cows ranged from 0 to 405 , with a mean value of 178.4 (SD 125.7).

Following preprocessing of the microarray data, gene expression levels expressed as M-values (log differentialexpression ratio between sample tissue and a common reference (which is the pool of all tissues) were obtained for each tissue per cow. Of the 16,620 probes on the microarray that were found to be of good quality based on reannotation, gene expression measures for 13,234 unique genes remained after averaging the expression levels of genes represented by multiple probes (see Table 1 for a summary of the steps taken in the analysis and their results).

\section{Consensus Modules Identified From Coexpression Networks Among Tissue Pairs}

We first checked the microarray data to ensure that the genes and arrays that were selected for detailed analysis did not have excessive numbers of missing values. These checks revealed that there were no such issues for any of the tissue pairs under study: AM-DH, HC-DH, VH-DH, AM-HC, and AP-DH. The clustering of arrays per tissue based on Euclidian distance did not point to any outlier arrays. For network construction, we chose one-half of the total 13,234 unique genes on the array that had the most variable expression levels across cows showing different levels of EB. This choice followed by selecting only those genes shared between the tissues in each pair resulted in between 4,000 and 5,000 genes per analysis (see Materials and Methods section for details).
Because biological networks generally follow a power law and tend to be approximately scale free, we chose a soft-thresholding power $(\beta=12)$ that results in a network with an approximate scale-free topology. The genes in the network were clustered into modules based on their connectivity strengths with all other genes in the network. The hierarchical clustering dendrograms produced for the consensus module identification in each pair of tissues are given in Supplementary Figure S1 (available online at http://www.journalofdairy science.org/). In the applied WGCNA software tool, the consensus modules found in the coexpression networks between tissue pairs were labeled by colors. After excluding the grey module, which is used to hold all genes that do not clearly belong to any other module, the number of modules found in each tissue pair were as follows: AM-DH: 3, HC-DH: 10, VH-DH: 2, AM-HC: 8, and AP-DH: 23. The largest number of genes within the consensus modules was observed for the AP-DH pair $(\mathrm{n}=1,904)$. This was followed by the HC-DH pair at 846 , AM-HC pair at 843 , AM-DH pair at 225 , and VH-DH pair at 197. A comparison of the genes within the consensus modules in the tissue pairs that had the DH in common (AP-DH, AM-DH, HC-DH, and $\mathrm{VH}-\mathrm{DH}$ ) revealed that the largest number of genes were shared between the AP-DH and HC-DH pairs and that 59 genes were shared among all 4 pairs (Venn diagram provided in Supplementary Figure S2, available online at http://www.journalofdairyscience.org/). The major biological processes enriched within these 59 shared genes were linked to the terms "ribosome" and "translation".

Table 2. Estrous behavior expressed as heat scores

\begin{tabular}{lc}
\hline d-0 cow no. & Heat score \\
\hline d0_5006 & 0 \\
d0_9284 & 37 \\
d0_3739 & 43 \\
d0_8855 & 53 \\
d0_1194 & 137 \\
d0_1821 & 175 \\
d0_8870 & 191 \\
d0_5507 & 200 \\
d0_1786 & 206 \\
d0_3472 & 246 \\
d0_6487 & 248 \\
d0_3747 & 378 \\
d0_7008 & 405 \\
d0_5125 & 1,750 \\
\hline
\end{tabular}

${ }^{1}$ For the analysis done here, heat score of this cow was replaced by that of the next-highest-scoring cow (i.e. score of 405) due to its unrealistically high outlier value. 
Table 3. Consensus modules whose module eigengenes correlated with estrous behavior ${ }^{1}$

\begin{tabular}{|c|c|c|c|c|}
\hline \multirow{2}{*}{$\frac{\text { Module }}{\text { AM-DH }}$} & \multirow[t]{2}{*}{$n^{2}$} & \multicolumn{2}{|c|}{$\begin{array}{c}\text { Correlation }\left(\begin{array}{c}P \text {-value }) \text { with estrous } \\
\text { behavior }\end{array}\right.\end{array}$} & \multirow[t]{2}{*}{ Top 3 hub genes } \\
\hline & & $\mathrm{AM}$ & $\mathrm{DH}$ & \\
\hline 1. Brown & 40 & $0.14(0.65)$ & $0.4(0.16) \dagger$ & OLFM1, NEFL, LY6E \\
\hline HC-DH & & $\mathrm{HC}$ & $\mathrm{DH}$ & \\
\hline 1. Magenta & 31 & $0.47(0.093) \dagger$ & $-0.3(0.31)$ & SLC25A47, TP53I3, CRYAA \\
\hline 2. Red & 51 & $0.62(0.016) \dagger$ & $0.014(0.96)$ & MATN1, MCM9, MTR \\
\hline 3. Black & 45 & $0.49(0.078) \dagger$ & $0.026(0.93)$ & CENPI, EXOSC1, DEPDC7 \\
\hline 4. Blue & 152 & $0.47(0.089) \dagger$ & $0.32(0.28)$ & RPL13A, RPS20, RPL18 \\
\hline 5. Green & 59 & $0.55(0.039) \dagger$ & $0.26(0.37)$ & CEP63, KRTAP13-1, AQP3 \\
\hline $\mathrm{AM}-\mathrm{HC}$ & & AM & $\mathrm{HC}$ & \\
\hline 1. Red & 52 & $-0.052(0.86)$ & $0.48(0.081) \dagger$ & NUP160, CCL21, NCAPG2 \\
\hline 2. Pink & 34 & $-0.33(0.25)$ & $0.47(0.091) \dagger$ & MED22, GBA, TP53I3 \\
\hline 3. Green & 77 & $0.32(0.28)$ & $0.42(0.14) \dagger$ & RPL18, HSPA8, RPS17 \\
\hline AP-DH & & $\mathrm{AP}$ & $\mathrm{DH}$ & \\
\hline 1. Green yellow & 74 & $0.42(0.14) \dagger$ & $0.0098(0.97)$ & NGEF, FAM 5C, C1QTNF5 \\
\hline 2. Midnight blue & 58 & $0.4(0.15) \dagger$ & $0.37(0.2)$ & NEFL, VSNL1, NDRG2 \\
\hline 3. Salmon & 71 & $0.4(0.16) \dagger$ & $0.35(0.23)$ & RNF128, PODNL1, HS3ST6 \\
\hline 4. Black & 88 & $0.4(0.16) \dagger$ & $0.26(0.37)$ & ENTPD $6, M G C 134087$, PHF13 \\
\hline 5. Purple & 77 & $0.39(0.17) \dagger$ & $0.26(0.37)$ & MRPL4O, HSCB, POLR2B \\
\hline 6. Turquoise & 343 & $-0.38(0.18) \dagger$ & $0.22(0.45)$ & SPG11, FRS2, GPC3 \\
\hline 7. Magenta & 84 & $-0.42(0.14) \dagger$ & $0.17(0.57)$ & KBTBD10,FNDC3A,IBSP \\
\hline 8. Brown & 114 & $-0.39(0.18) \dagger$ & $-0.13(0.65)$ & CHEK1, CBLL1, FANK1 \\
\hline 9. Pink & 86 & $-0.34(0.25)$ & $-0.39(0.18) \dagger$ & NPY2R, A5PK81, TMEM107 \\
\hline 10. Light cyan & 54 & $-0.38(0.19) \dagger$ & $0.09(0.77)$ & $M A N 2 C 1, T R D N, S L C 16 A 11$ \\
\hline
\end{tabular}

${ }^{1}$ Tissues are abbreviated as follows: anterior pituitary (AP), dorsal hypothalamus (DH), ventral hypothalamus (VH), amygdala (AM), and hippocampus (HC). The consensus modules (labeled by colors) in the coexpression networks between tissue pairs whose module eigengenes correlated with estrous behavior scores are presented here. Modules with an absolute correlation above 0.3 and having a P-value (in parentheses) below 0.2 are indicated by a $\dagger$ sign. The top 3 hub genes within these modules based on their intramodular connectivity measures are also presented. The VH-DH pair did not have any estrous behavior-correlated consensus modules.

${ }^{2}$ The number of genes found in each module.

\section{Consensus Modules Correlated With Estrous Behavior}

The consensus modules in the coexpression networks between tissue pairs that correlated with EB were detected based on the correlation between the module eigengene (weighted average expression profile of all genes within the module) and the heat scores. An absolute correlation above 0.3 was considered significant when the corresponding $P$-value was below 0.2 . The consensus modules that correlated with EB are presented in Table 3 along with the corresponding correlations and $P$-values per tissue. A module may be found significant in one tissue of the pair but not in the other. This difference in the correlation between the EB scores and module eigengenes for the same module between the individual tissues of a pair is due to the fact that the gene expression profiles per gene are not exactly the same between the paired tissues. The highest number of significant modules was found in the AP-DH pair (i.e., 10), whereas the VH-DH pair had none. The AM-DH pair had only 1 significant module, the $\mathrm{HC}-\mathrm{DH}$ pair had $5(P<0.1)$, and the AM-HC had $4(P<0.1)$. Also presented in Table 3 are the top 3 hub genes within each EB correlated module (i.e., the most highly connected genes in each module based on their module eigengene based kME). The complete list of all genes in all the EB-correlated consensus modules, ordered by their kME measures, is presented in Supplementary Table S1 (available online at http://www.journalofdairyscience. org/). Also presented there are the corresponding gene significance (GS) values, which are a measure of the correlation of that gene's expression with EB across all samples per tissue pair.

Tests for enriched gene ontology biological process (GOBP) terms and KEGG pathway terms in the EB-correlated modules revealed significant terms (Benjamini-Hochberg corrected $P$-value: $<0.15)$ in several modules in all the tissue pairs except the VH-DH. The results are summarized in Figure 1, whereas the full list can be found in Supplementary Table S2 (available online at http://www.journalofdairyscience.org/). Some of the key GOBP terms found in both AM-DH and HC-DH pairs were translation, biosynthetic process, and glial cell development. The terms translation and biosynthetic process were also found in the AM-HC pair but, in addition, there were terms such reproduction, intracellular signaling cascade, and production of molecular mediator of immune response. In all 3 pairs, the KEGG terms included one or more of the following: 


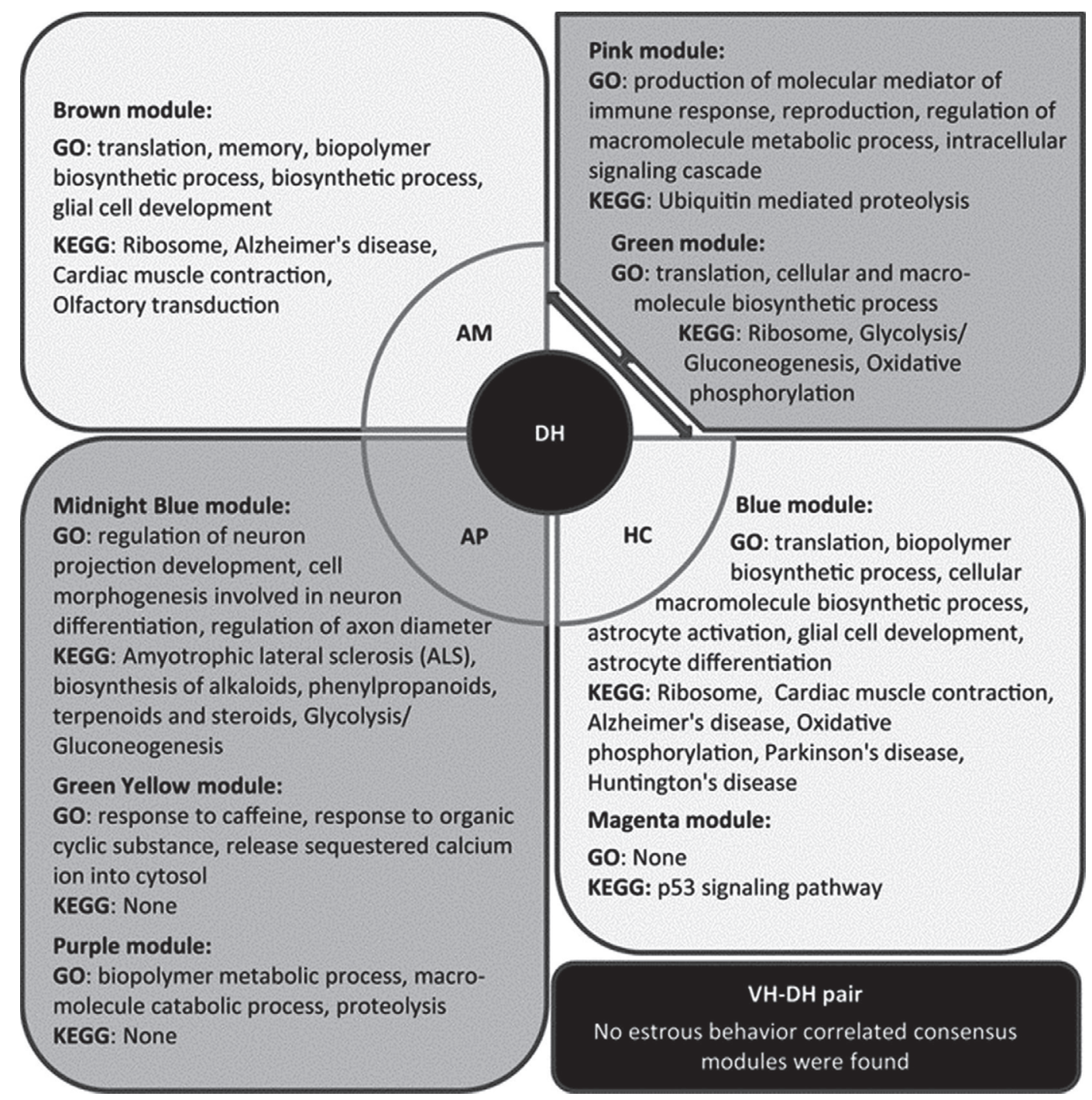

Figure 1. Biological processes within consensus modules among tissue pairs that significantly correlated with estrous behavior. The genes in the consensus modules found in coexpression networks between tissue pairs that significantly correlated with estrous behavior scores (absolute correlation $>0.3$ and $P<0.2$ ) were tested for overrepresented gene ontology biological process (GOBP) and Kyoto Encyclopedia of Genes and Genomes (KEGG) pathway terms. Those modules that showed significant GOBP and KEGG terms $(P<0.1)$ are presented here within boxes corresponding to each tissue pair. With the exception of the box in the upper right corner (representing amygdala-hippocampus pair), all others represent tissue pairs having the dorsal hypothalamus in common. $\mathrm{AM}=$ amygdala; $\mathrm{AP}=$ anterior pituitary; $\mathrm{HC}=$ hippocampus; $\mathrm{DH}=$ dorsal hypothalamus; $\mathrm{VH}=$ ventral hypothalamus.

ribosome, oxidative phosphorylation, and Alzheimer's disease. The terms enriched in the AP-DH pair shared less with the other tissue pairs and included GOBP terms such as response to caffeine, release of calcium ion, and regulation of neuron projection development, regulation of axon diameter, and neuron differentiation. The top KEGG terms found here were amyotrophic lateral sclerosis, biosynthesis of alkaloids and glycolysis/ gluconeogenesis. The "midnight blue" module in the AP-DH pair was especially interesting, as it showed several nervous system-related processes. From the biological processes found enriched in the different con- sensus modules in the 5 tissue pairs, it seems that processes specifically related to nervous system functioning are few, whereas the majority relates to general cellular processes. There were also several modules that did not reveal any significant GOBP or KEGG pathway terms.

As an example, we visualized as a network the "midnight blue" module found in the AP-DH (Figure 2). The nodes in this network correspond to genes in the module and the edges correspond to the intergenic connection strengths. It may be noted that most of the strongest connections are between genes that are also well connected with other genes (i.e., the hub genes). 


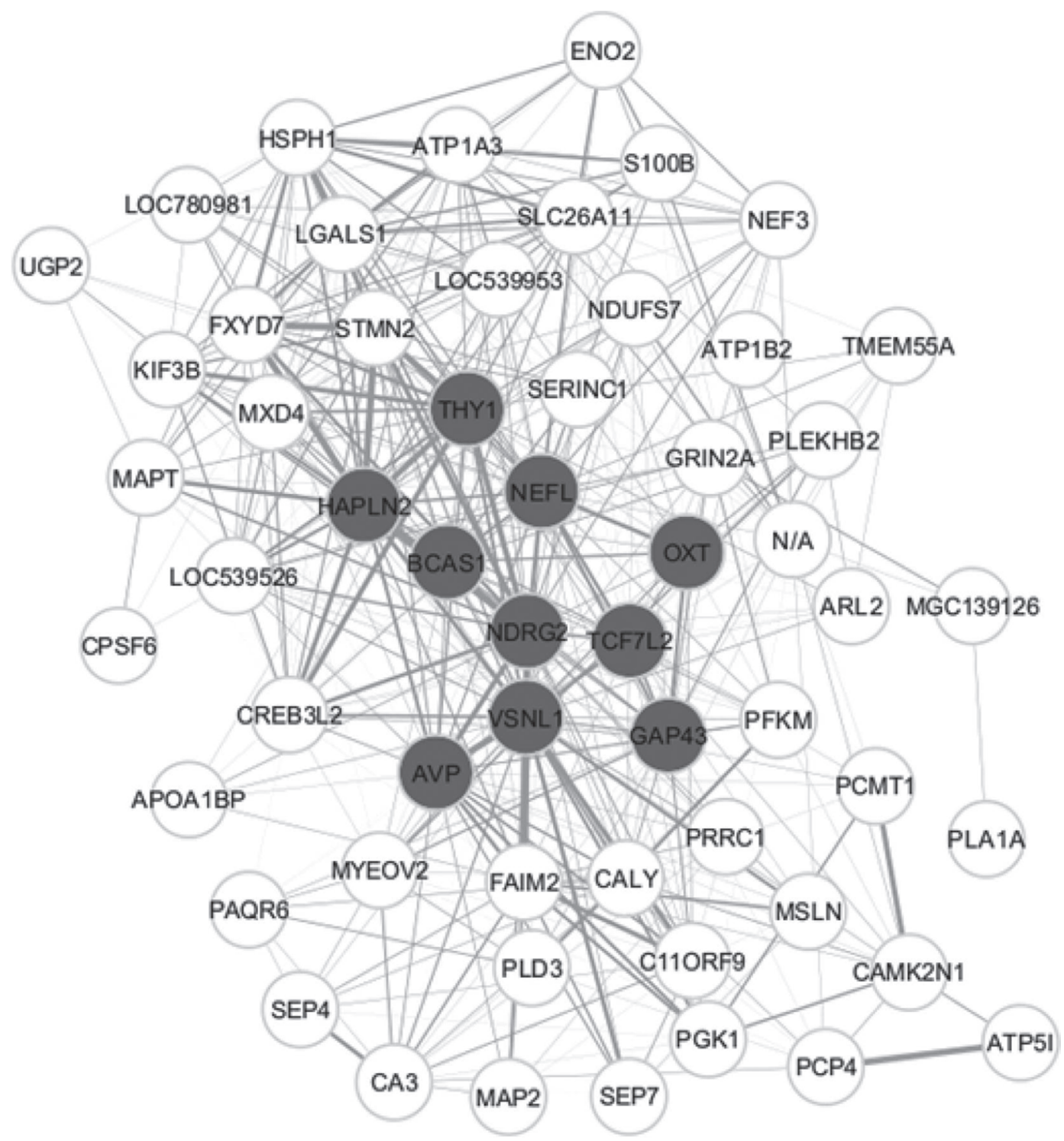

Figure 2. Network visualization of the "midnight blue" consensus module in the anterior pituitary-dorsal hypothalamus (AP-DH) pair. Nodes represent the genes in the module and edges represent the intergenic connection strengths. The 10 nodes with the highest intramodular connectivity measures (kME) are shaded. In general, nodes with higher kME values are placed towards the center of the network and have higher intergenic connection strengths. $N E F L=$ neurofilament, light polypeptide; $V S N L 1=$ visinin-like 1; $N D R G 2=\mathrm{N}$-myc downstream-regulated gene 2; $A V P=\operatorname{arginine}$ vasopressin; GAP 43 = growth-associated protein $43 ; B C A S 1=$ breast carcinoma amplified sequence $1 ; H A P L N 2=$ hyaluronan and proteoglycan link protein 2; THY1 = Thy-1 cell surface antigen; $O X T=$ oxytocin, prepropeptide; TCF $7 L 2=$ transcription factor 7 -like 2 .

The kME measures may be used to prioritize candidate genes for further in-depth studies and validation. The top 10 hub genes in the "midnight blue" network (shaded nodes, Figure 2) were as follows: neurofilament, light polypeptide $(N E F L)$, visinin-like 1 (VSNL1), N-myc downstream-regulated gene 2 (NDRG2), arginine vasopressin $(A V P)$, growth-associated protein 43 (GAP43), breast carcinoma amplified sequence 1 (BCAS1), hyaluronan and proteoglycan link protein 2 (HAPLN2), Thy-1 cell surface antigen (THY1), oxytocin, prepropeptide $(O X T)$, and transcription factor 7-like 2 (TCFYL2). The known functions of these hub genes indicated that they could be likely candidates for EB regulation. These genes are discussed in detail in the following section.

\section{DISCUSSION}

The objective of this study was to identify genes and biological processes shared among the bovine AP and brain areas that affect EB by investigating networks of coexpressed genes between these tissues. For this, 
we used a systems biology approach called WGCNA. The gene sets or modules thus identified were based on unsupervised clustering and not a priori defined. The genes in a module may have a common function, which can be ascertained based on pathway or gene ontology enrichment tests. Further, the most connected genes within a module (i.e. the hub genes) may indicate the most important genes that drive the process regulated by the genes in the module. WGCNA has been used successfully in a wide variety of studies to identify gene coexpression modules and genes within them that relate either to a trait or disease condition of interest (Ghazalpour et al., 2006; Horvath et al., 2006; Fuller et al., 2007; Miller et al., 2008) or that reflect underlying cellular composition or function of a tissue (Oldham et al., 2008; de Jong et al., 2010) or that are conserved between species to help understand aspects of tissue evolution (Oldham et al., 2006). One of the strengths of this method is that the problem of multiple testing corrections is eliminated. This is because here the correlation is between the trait and the module eigengene and not to individual gene expression levels. Further, studying gene coexpression promises new insights by suggesting that the coexpressed genes may be responding to common transcriptional regulatory factors or signals even in different tissues.

\section{Key Biological Processes Shared Between Brain Areas and AP That Are Associated With EB}

The highest number of consensus modules and genes within them that correlated with $\mathrm{EB}$ were found in the AP-DH pair. This is a reflection of the fact that the $\mathrm{AP}$ and DH play roles as centers in the hypothalamicpituitary-ovarian axis that regulate reproduction and fertility via the sex hormones. Also, the high number of consensus modules may indicate multiple biological processes that are either associated with the physiological state of estrus or that coordinate the regulation of the female reproductive cycle via a high level of communication between these areas and the ovary and via responding to specific signals, especially estrogen.

The fact that a number of modules correlate with EB may indicate that several genes and biological processes across several brain areas and the AP work together in the regulatory mechanism for EB expression. Knowledge of the components in this regulatory mechanism will help improve our understanding of EB regulation. Overrepresented GOBP terms in modules significantly correlated with EB included general brain processes like glial cell development, memory, astrocyte activation, regulation of neuron projection development, axon diameter and neurogenesis, or neurodegenerative diseases like Alzheimer's and Parkinson's diseases. Sev- eral of these processes were also found enriched among genes found associated with EB in our earlier studies at individual tissue level (Kommadath et al., 2010, 2011). From the current coexpression study, it becomes evident that these are important processes shared among the AP and brain areas and hints at a coordinated regulation via regulatory factors or signals across these tissues. We also found genes involved in general cellular processes like oxidative phosphorylation, glycolysis/gluconeogenesis, and ribosome. These general processes have been classified as general constitutive or housekeeping processes in several tissues (Prieto et al., 2008) and these have also been recognized as principal pathways in the brain (Gan et al., 2011; Marques et al., 2011) to meet its high energy demands. However, these processes are also enhanced during stress and vary with the stage of estrous cycle. Our finding of several ribosomal genes coexpressed in different pairs of tissues analyzed indicates a coordinated increase in ribosomal RNA synthesis. The enriched terms found like "ribosome," "oxidative phosphorylation," "biosynthetic processes," and so on indicate an increased rate of transcription and protein synthesis in the neuronal tissues at the start of estrus. This is supported by the following studies. It has been shown that progesterone and estrogen increase the oxidative capacity of wholebrain mitochondria and the activity of cytochrome c oxidase, a major mitochondrial enzyme responsible for oxidative respiration (Irwin et al., 2008). Further, a study of the cerebral cortex in female rats at different stages of the estrous cycle indicated a significant increase in the oxidative metabolism of the posterior cortex in the estrus and proestrus phases compared with the diestrus phase (Menéndez-Patterson et al., 1976). The increased synthesis of ribosomal RNA in the ventromedial hypothalamus has been reported to be one of the early effects of estrogen administered subcutaneously in ovariectomized rats and represents a primary event in the activation of neuronal cells and neuronal pathways involved in female reproductive behavior (Jones et al., 1990). Estrogen-driven expansion of dendrites and synapses follows from the stimulation of ribosomal RNA synthesis (Mong and Pfaff, 2004; Pfaff, 2005). Therefore, similar processes as identified in rodent studies also operate in cows to affect EB.

\section{Key Genes Driving Biological Processes Associated With EB}

The genes with the highest intramodular connectivity per module (i.e., hub genes) are generally the most important in the network and are supposed to be key regulators. Studying these genes can reveal the biological process driven by modules in the network. We found 
that the hub genes in some modules (e.g., "midnight blue" module) had a common function related to behavior regulation, nervous system processes, or energy metabolism, while in other modules, a common function could not be ascertained for the hub genes. In the latter case, it may be an indication that these genes are involved in some common hitherto unassigned function. We take here the example of the hub genes in the "midnight blue" module found in the AP-DH pair. Several of the top 10 hub genes in this module are known to have functions that could explain their role in EB regulation. For example, $N E F L$ is known to encode the light-chain neurofilament protein comprising the axoskeleton and thereby functionally maintain the neuronal caliber, and also plays a role in intracellular transport to axons and dendrites (Entrez gene summary for NEFL at http:// www.ncbi.nlm.nih.gov/gene/4747). The role of $N E F L$ in protein phosphatase-1 targeting to neuronal membranes and cytoskeleton and its potential for regulating the dephosphorylation of phosphoproteins implicated in synaptic plasticity has been shown (Terry-Lorenzo et al., 2000). Other hub genes involved in neuronal growth or plasticity include NDRG2 (Takahashi et al., 2005), THY1 (Rege and Hagood, 2006), and GAP43 (Gispen et al., 1991). Another hub gene, TCF $7 L 2$, encodes a high-mobility group (HMG) box-containing transcription factor (Castrop et al., 1992) and is an important effector in the Wnt signaling pathway (Jin and Liu, 2008), which is active in the development of the bloodbrain barrier, whereas the gene VSNL1 is a neuronal calcium sensor that has recently been identified as part of a signaling complex associated with purinergic receptors (Chaumont et al., 2008). These functions may link to findings of our earlier studies (Kommadath et al., 2010, 2011) where we concluded that synaptic plasticity is of importance for proper expression of EB. Further, hub genes such as $O X T$ and $A V P$, which encode the pituitary hormones oxytocin and arginine vasopressin, were also found in our earlier studies (Kommadath et al., 2010, 2011) as key genes whose expressions within individual tissues were correlated with $\mathrm{EB}$ and are known from the literature to have important regulatory functions related to socio-sexual behavior (Curley and Keverne, 2005; Donaldson and Young, 2008; Leng et al., 2008).

\section{Integrating Our Findings Within the Framework of the Growth, Amplification, Preparation, Permission, and Synchronization Modules}

Our findings in this study complement those from our previous studies to improve our overall understanding of the genomic regulation of EB in dairy cows. We now integrate these findings within the framework of the growth, amplification, preparation, permission, and synchronization (GAPPS) modules (Mong et al., 2003; Mong and Pfaff, 2004), a summary of which is provided in Table 4 (adapted from the PhD thesis of Kommadath, 2012).

In the following sentences, we first define what each of the GAPPS modules stands for as given by Mong et al. (2003) and Mong and Pfaff (2004) and then describe how our findings from this and our previous studies (Kommadath et al., 2010, 2011; Kommadath, 2012) fit in within each module. The GAPPS modules represent a set of 5 modules, based mainly on studies in rodents, to help explain the causal relations between sex hormones and female mating behavior. The first of these modules is the hormone-dependent growth response, driven by estrogen and progesterone, which triggers an increase in the input and output connections for behavior-directing hypothalamic neurons. This may be considered equivalent to processes related to synaptic plasticity found in this study and in our previous study (Kommadath et al., 2011). The second module is the amplification of the estrogen effect by progesterone, mediated by nuclear progesterone receptor. Though the progesterone receptor was not found coexpressed in the tissue pairs studied here, it was found upregulated in the AP at start of estrus compared to the middle of the estrous cycle (Kommadath, 2012). Next, the preparation module in GAPPS refers to preparation of the female for mating, which involves analgesia, anxiety reduction, social recognition, and aggression. Genes such as $O X T$ and $A V P$, found here as hub genes and also in our previous studies (Kommadath et al., 2010, 2011) as associated with heat scores, are known to play important roles in processes that help prepare the female for mating. The fourth module called permission refers to the permissive actions by hypothalamic neurons for the mating behavior to occur and the last module is the synchronization of mating behavior with ovulation. Several genes involved in both these modules have also been identified and discussed in our previous studies (Kommadath et al., 2010, 2011).

\section{Limitations and Future Direction of Research}

The relatively low number of animals in a networkbased study affects the statistical significance of the relationships found here. Despite this, the moderate correlation levels and the fact that many of the genes found here have (known) biological relevance to the trait under study indicates that even with a relatively high $P$-value cutoff of 0.2 , chosen to identify consensus modules that correlated with $\mathrm{EB}$, the resulting genes may be considered as candidates regulating EB. It should be noted that the products of some of the genes 
Table 4. Estrous behavior-associated genes and processes in dairy cows known to correspond to processes within the growth, amplification, preparation, permission, and synchronization (GAPPS) modules

\begin{tabular}{|c|c|c|}
\hline GAPPS module & Characteristics & $\begin{array}{l}\text { Corresponding genes and processes in cows } \\
\text { (with reference in parentheses) }\end{array}$ \\
\hline Growth & $\begin{array}{l}\text { Increase in the input/output connections for } \\
\text { behavior-directing hypothalamic neurons }\end{array}$ & $\begin{array}{l}\text { Synaptic plasticity: } \\
\text { Immune related genes: CTLA4, IL1RL1, MARCO (Kommadath et al., } \\
\text { 2011) } \\
\text { Neurotransmitter receptors: } C H R M 1, C H R M 3, \text { CHRNA5 (Kommadath et } \\
\text { al., 2011) } \\
\text { Ribosomal genes: RPL14, RPL18, RPL24, RPS11, RPS18 (this study) } \\
\text { Others: NEFL, NDRG2, THY1, GAP43 (this study) }\end{array}$ \\
\hline
\end{tabular}

Amplification Amplification of estrogen effect by progesterone mediated by progesterone receptor

Preparation Preparation for mating

Permission

Permissive actions by hypothalamic neurons for the mating behavior to occur

Synchronization Synchronize mating behavior with ovulation

PGR gene upregulated in the anterior pituitary at d 0 (Kommadath, 2012)

Female sexual receptivity: OXT, AVP, HTR2A, DRD2, GABRA6 (this study and Kommadath et al., 2011)

Anxiolytic effect: $O X T, T T R, K C N N 2$ (this study and Kommadath et al., 2011)

Altered feeding motivation and mood: POMC, MCHR1, MOBP, LTA4H

(Kommadath et al., 2011)

Arousal, activation of protein kinases and release of $\mathrm{Ca}^{2+}$ : CHRM1, CHRM3, CHRNA5, PLCB2, ITPKA (Kommadath et al., 2011)

Prostaglandin regulators: PTGDS, PTGIS, PTGFR (Kommadath et al., 2011)

${ }^{1}$ This table is adapted from the PhD thesis of the first author (Kommadath, 2012).

within the coexpressed modules may fall in different cellular compartments and may have no possibility for physical interaction in vivo. Nevertheless, their belonging together in a module may indicate that the coexpressed genes across tissue pairs may be responding to specific regulatory factors or signals. Thereby new hypotheses may be formulated on how the expression of genes in the AP and brain areas is coordinated to function together in regulating the trait of $\mathrm{EB}$. To make this knowledge practically useful in dairy farming, it is important to first validate the most promising candidate genes identified here, especially the genes also known from the literature to have functions related to EB in other species. How the validation can be achieved would, however, be a challenge, given the difficulty of collecting brain tissue samples from cows during their lifetime. Use of laboratory animals like rodents will allow the use of selective knock-out or knock-down mutants to study the influence of genes identified in our study. There is also the possibility of using advanced mathematical models to assist in the validation of the roles of these genes. The use of mathematical models for the estrous cycle in cows with inputs from physiological parameters has been reported (Boer et al., 2011). It would be valuable to extend the latter model to study how changes in expression of key genes identified in our study affect EB expression. A recent study showed that though transcriptome organization was generally poorly preserved between brain and blood, there were certain genes in the consensus modules, especially those involved in basic cellular processes, which exhibited strong preservation (Cai et al., 2010). Therefore in future, the correlation between the level of a protein or metabolite secreted in the milk or blood and the gene expression profile of genes found correlated with EB here may be studied. Highly correlated cases may be potential biomarkers, which could be considered for development of quick assays to determine the estrous status of the cow. Biomarkers for estrus may also be developed from EB-correlated gene products from the brain that cross the blood-brain barrier or those from the anterior pituitary, which can potentially be measured in the blood.

\section{CONCLUSIONS}

To conclude, this study has revealed important new aspects of the genomic regulation of EB in dairy cows. Key findings of this study have been integrated with findings of our earlier studies to present a summary of the genomic regulation of $\mathrm{EB}$ in dairy cows within the framework of the GAPPS modules initially proposed in rodents. Our findings would serve as a valuable reference to provide leads on genes to follow on in future research on understanding EB regulation. The new knowledge could ultimately be used to optimize fertil- 
ity of dairy cows by aiding the improvement of existing tools or help in the development of novel reproductive management tools such as diagnostic tools to determine the reproductive health, energy, and fertility status of the cow, estrus-detection tools, and so on.

\section{ACKNOWLEDGMENTS}

We thank Roel Veerkamp (Animal Breeding and Genomics Centre, Wageningen UR Livestock Research, Lelystad, the Netherlands) and Martien Groenen (Animal Breeding and Genomics Centre, Wageningen University) for helpful discussions and suggestions to improve the manuscript. This work was co-financed by the European Commission-funded projects SABRE (http://www.sabre-eu.eu/; contract no. FOODCT-2006-016250) and EADGENE (http://www. eadgene.info/; contract no. FOOD-CT-2004-506416); the Dutch Ministry of Agriculture, Nature and Food Quality (http://www.minlnv.nl; KB-04-004-012); and the international cattle breeding cooperative CRV BV (http://www.crv4all.com/). The text represents the authors' views and does not necessarily represent a position of the Commission who will not be liable for the use made of such information.

\section{REFERENCES}

Ashburner, M., C. A. Ball, J. A. Blake, D. Botstein, H. Butler, J. M Cherry, A. P. Davis, K. Dolinski, S. S. Dwight, J. T. Eppig, M. A. Harris, D. P. Hill, L. Issel-Tarver, A. Kasarskis, S. Lewis, J. C. Matese, J. E. Richardson, M. Ringwald, G. M. Rubin, and G. Sherlock. 2000. Gene ontology: Tool for the unification of biology. Nat. Genet. 25:25-29.

Bauersachs, S., S. E. Ulbrich, K. Gross, S. E. M. Schmidt, H. H. D. Meyer, R. Einspanier, H. Wenigerkind, M. Vermehren, H. Blum, F. Sinowatz, and E. Wolf. 2005. Gene expression profiling of bovine endometrium during the oestrous cycle: Detection of molecular pathways involved in functional changes. J. Mol. Endocrinol. 34:889-908.

Benjamini, Y., and Y. Hochberg. 1995. Controlling the false discovery rate: A practical and powerful approach to multiple testing. J. R. Stat. Soc., B 57:289-300.

Boer, H. M. T., C. Stötzel, S. Röblitz, P. Deuflhard, R. F. Veerkamp, and $\mathrm{H}$. Woelders. 2011. A simple mathematical model of the bovine estrous cycle: Follicle development and endocrine interactions. J. Theor. Biol. 278:20-31.

Butler, W. R. 2000. Nutritional interactions with reproductive performance in dairy cattle. Anim. Reprod. Sci. 60-61:449-457.

Cai, C., P. Langfelder, T. Fuller, M. Oldham, R. Luo, L. van den Berg, R. Ophoff, and S. Horvath. 2010. Is human blood a good surrogate for brain tissue in transcriptional studies? BMC Genomics 11:589.

Carlson, M. 2011. org.Bt.eg.db: Genome wide annotation for Bovine. $\mathrm{R}$ package version 2.8.0. Accessed June 2011. http://www. bioconductor.org/packages/2.8/data/annotation/html/org.Bt.eg. db.html.

Casel, P., F. Moreews, S. Lagarrigue, and C. Klopp. 2009. sigReannot: an oligo-set re-annotation pipeline based on similarities with the Ensembl transcripts and Unigene clusters. BMC Proc. 3:S3.

Casey, O. M., D. G. Morris, R. Powell, J. M. Sreenan, and R. Fitzpatrick. 2005. Analysis of gene expression in non-regressed and regressed bovine corpus luteum tissue using a customized ovarian cDNA array. Theriogenology 64:1963-1976.

Castrop, J., K. van Norren, and H. Clevers. 1992. A gene family of HMG-box transcription factors with homology to TCF-1. Nucleic Acids Res. 20:611.

Chaumont, S., V. Compan, E. Toulme, E. Richler, G. D. Housley, F. Rassendren, and B. S. Khakh. 2008. Regulation of P2X2 receptors by the neuronal calcium sensor VILIP1. Sci. Signal. 1:ra8.

Curley, J. P., and E. B. Keverne. 2005. Genes, brains and mammalian social bonds. Trends Ecol. Evol. 20:561-567.

de Jong, S., T. Fuller, E. Janson, E. Strengman, S. Horvath, M. Kas, and R. Ophoff. 2010. Gene expression profiling in C57BL/6J and $\mathrm{A} / \mathrm{J}$ mouse inbred strains reveals gene networks specific for brain regions independent of genetic background. BMC Genomics 11:20.

Donaldson, Z. R., and L. J. Young. 2008. Oxytocin, vasopressin, and the neurogenetics of Sociality. Science 322:900-904.

Eisen, M. B., P. T. Spellman, P. O. Brown, and D. Botstein. 1998 Cluster analysis and display of genome-wide expression patterns. Proc. Natl. Acad. Sci. USA 95:14863-14868.

Falcon, S., and R. Gentleman. 2007. Using GOstats to test gene lists for GO term association. Bioinformatics 23:257-258.

Fuller, T., A. Ghazalpour, J. E. Aten, T. A. Drake, A. J. Lusis, and S. Horvath. 2007. Weighted gene coexpression network analysis strategies applied to mouse weight. Mamm. Genome 6:463-472.

Gallagher, M., and A. A. Chiba. 1996. The amygdala and emotion. Curr. Opin. Neurobiol. 6:221-227.

Gan, L., X. Liu, Z. Xiang, and N. He. 2011. Microarray-based gene expression profiles of silkworm brains. BMC Neurosci. 12:8.

Garnsworthy, P. C., K. D. Sinclair, and R. Webb. 2008. Integration of physiological mechanisms that influence fertility in dairy cows. Animal 2:1144-1152.

Gentleman, R. C., V. J. Carey, D. M. Bates, B. Bolstad, M. Dettling, S. Dudoit, B. Ellis, L. Gautier, Y. Ge, J. Gentry, K. Hornik, T. Hothorn, W. Huber, S. Iacus, R. Irizarry, F. Leisch, C. Li, M. Maechler, A. J. Rossini, G. Sawitzki, C. Smith, G. Smyth, L. Tierney, J. Y. H. Yang, and J. Zhang. 2004. Bioconductor: Open software development for computational biology and bioinformatics. Genome Biol. 5:R80.

Ghazalpour, A., S. Doss, B. Zhang, S. Wang, C. Plaisier, R. Castellanos, A. Brozell, E. E. Schadt, T. A. Drake, A. J. Lusis, and S. Horvath. 2006. Integrating genetics and network analysis to characterize genes related to mouse weight. PLoS Genet. 2:e130.

Gispen, W. H., H. B. Nielander, P. N. E. De Graan, A. B. Oestreicher, L. H. Schrama, and P. Schotman. 1991. Role of the growth-associated protein B-50/GAP-43 in neuronal plasticity. Mol. Neurobiol. 5:61-85.

Horvath, S., and J. Dong. 2008. Geometric interpretation of gene coexpression network analysis. PLoS Comput. Biol. 4:e1000117.

Horvath, S., B. Zhang, M. Carlson, K. V. Lu, S. Zhu, R. M. Felciano, M. F. Laurance, W. Zhao, S. Qi, Z. Chen, Y. Lee, A. C. Scheck, L. M. Liau, H. Wu, D. H. Geschwind, P. G. Febbo, H. I. Kornblum, T. F. Cloughesy, S. F. Nelson, and P. S. Mischel. 2006. Analysis of oncogenic signaling networks in glioblastoma identifies $A S P M$ as a novel molecular target. Proc. Natl. Acad. Sci. USA 103:17402-17407.

Huang, Y., H. Li, H. Hu, X. Yan, M. S. Waterman, H. Huang, and X. J. Zhou. 2007. Systematic discovery of functional modules and context-specific functional annotation of human genome. Bioinformatics 23:i222-i229.

Ideker, T., O. Ozier, B. Schwikowski, and A. F. Siegel. 2002. Discovering regulatory and signalling circuits in molecular interaction networks. Bioinformatics 18(Suppl. 1):S233-S240.

Irwin, R. W., J. Yao, R. T. Hamilton, E. Cadenas, R. D. Brinton, and J. Nilsen. 2008. Progesterone and estrogen regulate oxidative metabolism in brain mitochondria. Endocrinology 149:3167-3175.

Jin, T., and L. Liu. 2008. Minireview: The Wnt signaling pathway effector TCF7L2 and type 2 diabetes mellitus. Mol. Endocrinol. $22: 2383-2392$

Jones, K. J., C. A. Harrington, D. M. Chikaraishi, and D. W. Pfaff. 1990. Steroid hormone regulation of ribosomal RNA in rat hypo- 
thalamus: Early detection using in situ hybridization and precursor-product ribosomal DNA probes. J. Neurosci. 10:1513-1521.

Kanehisa, M., and S. Goto. 2000. KEGG: Kyoto Encyclopedia of Genes and Genomes. Nucleic Acids Res. 28:27-30.

Kommadath, A. 2012. Genomic regulation of oestrous behaviour in dairy cows. PhD Thesis. Wageningen University, Wageningen, the Netherlands.

Kommadath, A., H. A. Mulder, A. A. C. de Wit, H. Woelders, M. A. Smits, B. Beerda, R. F. Veerkamp, A. C. J. Frijters, and M. F. W. te Pas. 2010. Gene expression patterns in anterior pituitary associated with quantitative measure of oestrous behaviour in dairy cows. Animal 4:1297-1307.

Kommadath, A., H. Woelders, B. Beerda, H. A. Mulder, A. A. C. de Wit, R. F. Veerkamp, M. F. W. te Pas, and M. A. Smits. 2011. Gene expression patterns in four brain areas associate with quantitative measure of estrous behavior in dairy cows. BMC Genomics 12:200.

Langfelder, P., and S. Horvath. 2007. Eigengene networks for studying the relationships between co-expression modules. BMC Syst. Biol. 1:54

Langfelder, P., and S. Horvath. 2008. WGCNA: An R package for weighted correlation network analysis. BMC Bioinformatics 9:559.

Langfelder, P., B. Zhang, and S. Horvath. 2008. Defining clusters from a hierarchical cluster tree: The Dynamic Tree Cut package for $\mathrm{R}$. Bioinformatics 24:719-720.

Leng, G., S. L. Meddle, and A. J. Douglas. 2008. Oxytocin and the maternal brain. Curr. Opin. Pharmacol. 8:731-734.

Lopez, H., L. D. Satter, and M. C. Wiltbank. 2004. Relationship between level of milk production and estrous behavior of lactating dairy cows. Anim. Reprod. Sci. 81:209-223.

Marques, F., J. C. Sousa, G. Coppola, F. Gao, R. Puga, H. Brentani, D. H. Geschwind, N. Sousa, M. Correia-Neves, and J. A. Palha. 2011. Transcriptome signature of the adult mouse choroid plexus Fluids and Barriers of the CNS 8:10.

Menéndez-Patterson, A., J. A. Florez-Lozano, and B. Marin. 1976 Oxidative and glicolytic metabolism of the frontal cortex (laterofrontal) and of the posterior cortex (latero-occipital) in relation with the sexual activity of the rat. Reproduccion 3:279-285.

Miller, J. A., M. C. Oldham, and D. H. Geschwind. 2008. A systems level analysis of transcriptional changes in Alzheimer's disease and normal aging. J. Neurosci. 28:1410-1420.

Mong, J., A. Easton, L.-M. Kow, and D. Pfaff. 2003. Neural, hormonal and genetic mechanisms for the activation of brain and behavior. Eur. J. Pharmacol. 480:229-231.

Mong, J. A., and D. W. Pfaff. 2004. Hormonal symphony: Steroid orchestration of gene modules for sociosexual behaviors. Mol. Psychiatry 9:550-556.

Oldham, M. C., S. Horvath, and D. H. Geschwind. 2006. Conservation and evolution of gene coexpression networks in human and chimpanzee brains. Proc. Natl. Acad. Sci. USA 103:17973-17978.
Oldham, M. C., G. Konopka, K. Iwamoto, P. Langfelder, T. Kato, S. Horvath, and D. H. Geschwind. 2008. Functional organization of the transcriptome in human brain. Nat. Neurosci. 11:1271-1282.

Pfaff, D. 2005. Hormone-driven mechanisms in the central nervous system facilitate the analysis of mammalian behaviours. J. Endocrinol. 184:447-453.

Prieto, C., A. Risueño, C. Fontanillo, and J. De Las Rivas. 2008. Human gene coexpression landscape: Confident network derived from tissue transcriptomic profiles. PLoS ONE 3:e3911.

Rege, T. A., and J. S. Hagood. 2006. Thy-1 as a regulator of cell-cell and cell-matrix interactions in axon regeneration, apoptosis, adhesion, migration, cancer, and fibrosis. FASEB J. 20:1045-1054.

Salamon, E., T. Esch, and G. B. Stefano. 2005. Role of amygdala in mediating sexual and emotional behavior via coupled nitric oxide release. Acta Pharmacol. Sin. 26:389-395.

Salilew-Wondim, D., M. Hölker, F. Rings, N. Ghanem, M. Ulas-Cinar, J. Peippo, E. Tholen, C. Looft, K. Schellander, and D. Tesfaye. 2010. Bovine pretransfer endometrium and embryo transcriptome fingerprints as predictors of pregnancy success after embryo transfer. Physiol. Genomics 42:201-218.

Shannon, P., A. Markiel, O. Ozier, N. S. Baliga, J. T. Wang, D. Ramage, N. Amin. B. Schwikowski, and T. Ideker. 2003. Cytoscape: A software environment for integrated models of biomolecular interaction networks. Genome Res. 13:2498-2504.

Smyth, G. K. 2005. Limma: Linear models for microarray data. Pages 397-420 in Bioinformatics and Computational Biology Solutions using R and Bioconductor. R. Gentleman, V. Carey, S. Dudoit, R. Irizarry, and W. Huber, ed. Springer, New York, NY.

Stott, A. W., R. F. Veerkamp, and T. R. Wassell. 1999. The economics of fertility in the dairy herd. Anim. Sci. 68:49-57.

Takahashi, K., M. Yamada, H. Ohata, K. Honda, and M. Yamada. 2005. Ndrg2 promotes neurite outgrowth of NGF-differentiated PC12 cells. Neurosci. Lett. 388:157-162.

Terry-Lorenzo, R. T., M. Inoue, J. H. Connor, T. A. J. Haystead, B. N. Armbruster, R. P. Gupta, C. J. Oliver, and S. Shenolikar. 2000 Neurofilament-L is a protein phosphatase-1-binding protein associated with neuronal plasma membrane and post-synaptic density. J. Biol. Chem. 275:2439-2446.

Ushizawa, K., C. B. Herath, K. Kaneyama, S. Shiojima, A. Hirasawa, T. Takahashi, K. Imai, K. Ochiai, T. Tokunaga, Y. Tsunoda, G. Tsujimoto, and K. Hashizume. 2004. cDNA microarray analysis of bovine embryo gene expression profiles during the pre-implantation period. Reprod. Biol. Endocrinol. 2:77.

Zhang, B., and S. Horvath. 2005. A general framework for weighted gene co-expression network analysis. Stat. Appl. Genet. Mol. Biol. $4: 17$. 